\title{
Prevention of venous thromboembolism in patients undergoing bariatric surgery
}

\author{
This article was published in the following Dove Press journal: \\ Vascular Health and Risk Management \\ 17 August 2015 \\ Number of times this article has been viewed
}

\author{
Matthew A Bartlett \\ Karen F Mauck \\ Paul R Daniels \\ Division of General Internal Medicine, \\ Mayo Clinic Thrombophilia Center, \\ Department of Medicine, Mayo Clinic, \\ Rochester, MN, USA
}

\begin{abstract}
Bariatric surgical procedures are now a common method of obesity treatment with established effectiveness. Venous thromboembolism (VTE) events, which include deep vein thrombosis and pulmonary embolism, are an important source of postoperative morbidity and mortality among bariatric surgery patients. Due to an understanding of the frequency and seriousness of these complications, bariatric surgery patients typically receive some method of VTE prophylaxis with lower extremity compression, pharmacologic prophylaxis, or both. However, the optimal approach in these patients is unclear, with multiple open questions. In particular, strategies of adjusted-dose heparins, postdischarge anticoagulant prophylaxis, and the role of vena cava filters have been evaluated, but only to a limited extent. In contrast to other types of operations, the literature regarding VTE prophylaxis in bariatric surgery is notable for a dearth of prospective, randomized clinical trials, and current professional guidelines reflect the uncertainties in this literature. Herein, we summarize the available evidence after systematic review of the literature regarding approaches to VTE prevention in bariatric surgery. Identification of risk factors for VTE in the bariatric surgery population, analysis of the effectiveness of methods used for prophylaxis, and an overview of published guidelines are presented.
\end{abstract}

Keywords: bariatric surgery, venous thromboembolism, prophylaxis, vena cava filter, heparin

\section{Introduction}

Surgical approaches to weight loss, bariatric surgeries, are commonly performed procedures for morbidly obese individuals; the estimated number of bariatric procedures in the USA alone was close to 180,000 in 2013. Bariatric surgery is effective in achieving weight loss and improving obesity-related complications. ${ }^{1-3}$ However, there are also potential risks or complications, among them venous thromboembolism (VTE). Reported rates of VTE, including deep vein thrombosis (DVT) and pulmonary embolism (PE), following bariatric surgery are $0.3 \%-2.2 \%$, with rates of PE being approximately $1 \%$, despite application of methods to prevent these complications. ${ }^{4-9}$ $\mathrm{PE}$ is a frequent cause of postoperative mortality in the bariatric surgery population and is a common finding at autopsy. ${ }^{10-12}$

Various strategies have been used to prevent VTE in patients undergoing bariatric surgery, including pharmacologic and mechanical approaches. However, the optimal approach remains unclear. The objective of this review is to discuss and evaluate the existing literature regarding prevention of VTE in bariatric surgery patients. This review includes identification of risk factors for VTE in the bariatric surgery population,
Correspondence: Paul R Daniels Division of General internal Medicine, Mayo Clinic Thrombophilia Center, Department of Medicine, Mayo Clinic 200 First Street SW, Rochester, MN 55905, USA

Tel +l 5072661619

Fax +I 5072845370

Email daniels.paul@mayo.edu 
analysis of the effectiveness of methods used for prophylaxis, and an overview of published guidelines.

\section{Methods}

\section{Search strategy}

A systematic literature search was performed in MEDLINE (1946-January 12, 2015), EMBASE (*1947-January 12, 2015), Cochrane Database for Systematic Reviews, and Clinicaltrials.gov and is depicted in Table 1. The search was limited to English language studies. Search terms for bariatric surgery included both generic and specific terms for various bariatric procedures; broad terms for venous thrombosis and methods of prophylaxis were used.

\section{Study selection and data abstraction}

Inclusion and exclusion criteria to identify studies for this review are shown in Table 2 . The primary aim of these criteria was to identify studies which report outcomes from two or more treatment groups or include multivariate analysis to control for VTE risk factors. Abstracts not published as peer reviewed articles were not included.

Following the primary literature search, two reviewers (MB and PD) independently screened all articles to ensure satisfaction of inclusion/exclusion criteria. Reference lists of included articles were reviewed to identify additional publications of interest. Information on general study characteristics (study design, number of participants, study period, and follow-up), study participants (age, sex, body mass index $[\mathrm{BMI}])$, bariatric procedure type, methods of prophylaxis, and outcome measures was collected.

\section{Results of study selection}

A summary of the selected studies is presented in Tables 3 and 4 (Table 3: mechanical and pharmacologic prophylaxis studies; Table 4: vena cava filter [VCF] studies). Regarding study designs used, only two were prospective, randomized trials; all others were either retrospective or prospective cohort studies. Most studies were single center, although there were large database driven studies included. ${ }^{13-16}$ Sample sizes were relatively small with respect to numbers needed to detect differences in the primary outcome metrics (VTE). In some cohort studies, different treatment groups were recruited consecutively over several years. ${ }^{17-22}$

Modes of prophylaxis reported include lower extremity compression (LEC), anticoagulation therapy including subcutaneous (SC) low-molecular-weight heparins (LMWHs) and unfractionated heparin (UFH), and VCFs. Multiple studies addressed the question of adjusted-dose heparin. ${ }^{19,23-28} \mathrm{~A}$ few of the studies of pharmacologic prophylaxis included patients who also received a VCF, though the proportions with VCF were small. ${ }^{13,14,28}$

Roux-en-Y gastric bypass (RYGB), either laparoscopic or open, was the only procedure type in multiple studies. . $^{17,18,22,24,26,28-30}$ The proportion of open versus laparoscopic of any procedure type was not always reported. ${ }^{14,21,23,24,27,31}$ A few studies did not describe patient age, proportion of male participants, or BMI of study subjects. ${ }^{18,22,32}$ In some cases, patients with a history of VTE were excluded, and in some cases, the frequency of patients with

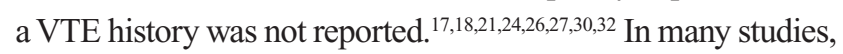
comparisons of the frequencies of patient VTE risk factors or of procedure type are not reported or tested. ${ }^{17,21,22,26,27,29-32}$

Table I Primary search strategy

\begin{tabular}{|c|c|c|}
\hline Number & Searches & Results \\
\hline I & exp Bariatric Surgery/ & 15,193 \\
\hline \multirow[t]{6}{*}{2} & ("bariatric operation*" or "bariatric procedure*" or "bariatric surg*" or "biliopancreatic & 19,977 \\
\hline & bypass*" or "biliopancreatic diversion*" or "duodenal switch*" or "gastric band*" or & \\
\hline & "gastric bypass*” or "gastroileal bypass*” or gastrojejunostom* or gastroplast* or & \\
\hline & "ileojejunal bypass*" or "intestinal bypass*" or "jejunoileal bypass*” or "jejuno-ileal & \\
\hline & bypass*" or "metabolic surg*" or "obesity surg*" or "pancreatobiliary bypass*" or "sleeve & \\
\hline & gastrectom*" or "stomach band*” or "stomach stapl*”).mp. & \\
\hline 3 & I or 2 & 22,652 \\
\hline 4 & exp Thromboembolism/pc & 10,713 \\
\hline 5 & exp venous thromboembolism/pc & 2,379 \\
\hline \multirow[t]{2}{*}{6} & ("deep thrombophlebitis" or "deep vein thromb*” or "deep venous thromb*” or & 83,653 \\
\hline & thromboembolism* or “pulmonary embol*”).mp. & \\
\hline \multirow[t]{2}{*}{7} & (anticoagulant* or heparin or filter* or compression or "venous foot pump*" or prevent* & $1,418,647$ \\
\hline & or prophyla*).mp. & \\
\hline 8 & 4 and (vein* or venous).mp. & 4,661 \\
\hline 9 & 3 and $(5$ or $(6$ and 7$)$ or 8$)$ & 222 \\
\hline 10 & Limit 9 to English language & 204 \\
\hline II & Remove duplicates from 10 & 203 \\
\hline
\end{tabular}


Table 2 Inclusion and exclusion criteria for identified publications

\begin{tabular}{ll}
\hline Inclusion criteria & Exclusion criteria \\
\hline - Human subjects & - Animal studies \\
- Patients undergoing bariatric surgery & - Study subjects $<18$ years of age \\
- Study designs: randomized trials, or cohort studies comparing & - Patients undergoing contouring and plastic surgery following bariatric surgery \\
two or more groups & \\
- Studies comparing VTE prophylaxis strategies: lower extremity & - Abstract only (no peer-reviewed published article) \\
compression, pharmacologic prophylaxis, or vena cava filters & \\
- Studies reporting postoperative clinical outcomes: venous & - Observational studies with no control or comparison group or study data \\
thromboembolism, bleeding complications, or mortality & presented without multivariate analysis \\
- English language only &
\end{tabular}

Abbreviation: VTE, venous thromboembolism.

All studies except one included in this review report VTE outcomes. ${ }^{28}$ In general, VTE events were identified based on testing directed to symptomatic patients or clinical suspicion of disease, although two studies performed imaging to detect asymptomatic DVT. ${ }^{20,23} \mathrm{PE}$ was diagnosed through evaluation of symptoms. Bleeding events are reported in studies in which the primary aim was evaluation of pharmacologic prophylaxis. However, there is no standard definition of bleeding severity across these reports, making comparisons challenging. The majority of studies report postoperative mortality, but do not always distinguish PE-related death from other causes. Filter-related complications are not consistently reported across the studies of VCF as prophylaxis..$^{15,22,33}$ The postoperative outcome ascertainment period for most studies was between 30 and 90 days.

\section{Risk factors forVTE among bariatric surgery patients}

Risk factors for postoperative VTE have been identified for surgical patients and have been incorporated into validated risk assessment tools. ${ }^{34,35}$ An examination of VTE risk factors specific to the bariatric surgery population is warranted; this understanding provides insight into the bariatric surgery prophylaxis literature and could refine future prevention strategies. Postoperative VTE risk factors can be categorized as patient related or procedure related; identified risk factors from at least one published report are presented in Table 5.

\section{Patient-related risk factors for VTE}

Among patient-related characteristics, multiple studies have found that the male sex is associated with an elevated risk of VTE among bariatric surgery patients..$^{7,9,36-38}$ Preoperative patient weight and BMI have also been associated with an increased risk of VTE events. ${ }^{7-9,39,40}$ For example, Finks et $\mathrm{al}^{9}$ demonstrated that every 10 unit increment in BMI was associated with a $37 \%$ increase in VTE risk (relative risk
[RR], 1.37; confidence interval [CI], 1.06-1.75). Several studies have also identified increasing age as a risk factor for postoperative VTE. ${ }^{7-9,38,41}$ Patient smoking status has been identified as a potential VTE risk factor in two reports. ${ }^{38,41}$ Several of the previously referenced studies have identified a prior history of VTE as a predictor of postoperative VTE in the bariatric surgery population..$^{7,938,41}$ For example, Finks et $\mathrm{al}^{9}$ demonstrated that a prior history of VTE was associated with four times the risk of postoperative VTE (odds ratio [OR], 4.15; CI, 2.42-7.08). Studies have also evaluated the presence of possible markers of hypercoagulability among bariatric surgery patients but have not assessed an association with clinical VTE. ${ }^{42-44}$

\section{Procedure-related risks factors for VTE}

Procedure-related factors for VTE after bariatric surgery include operative time, procedure type, postoperative complications, and whether the procedure is open or laparoscopic. In the bariatric surgery literature, studies have indicated an increased risk of VTE with open compared with laparoscopic procedures. ${ }^{7,836}$ Regarding duration of surgery, Finks et a ${ }^{9}$ reported an $86 \%$ increased risk of VTE with operative time $>3$ hours, (RR, 1.86; CI, 1.07-3.26). Chan et a ${ }^{45}$ also identified operative time $>3$ hours as an independent predictor of postoperative VTE and found that preoperative BMI was an independent predictor of operative time. In the analysis by Jamal et al, ${ }^{8}$ revision surgeries were associated with elevated VTE risk. The report by Gonzalez et $\mathrm{al}^{41}$ found that postoperative anastomotic leak after RYGB was also associated with increased VTE risk. In a comparison of different types of bariatric procedures, Finks et $\mathrm{al}^{9}$ found higher VTE risk with sleeve gastrectomy, laparoscopic gastric bypass, open RYGB, and duodenal switch surgery when compared to adjustable gastric band procedures. Masoomi et a ${ }^{136}$ found that gastric bypass procedures carry higher VTE risk when compared to other types of bariatric procedures. 


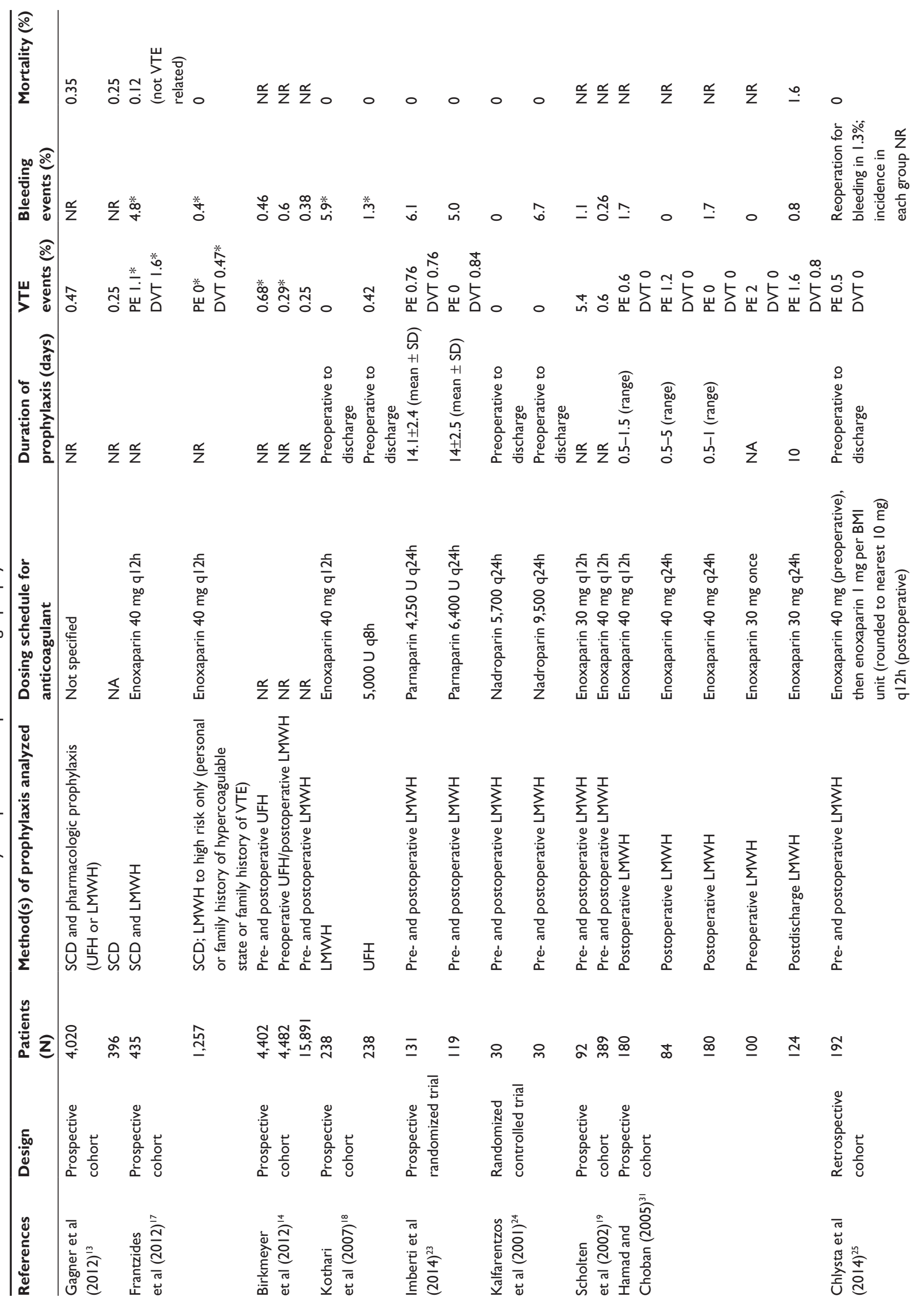



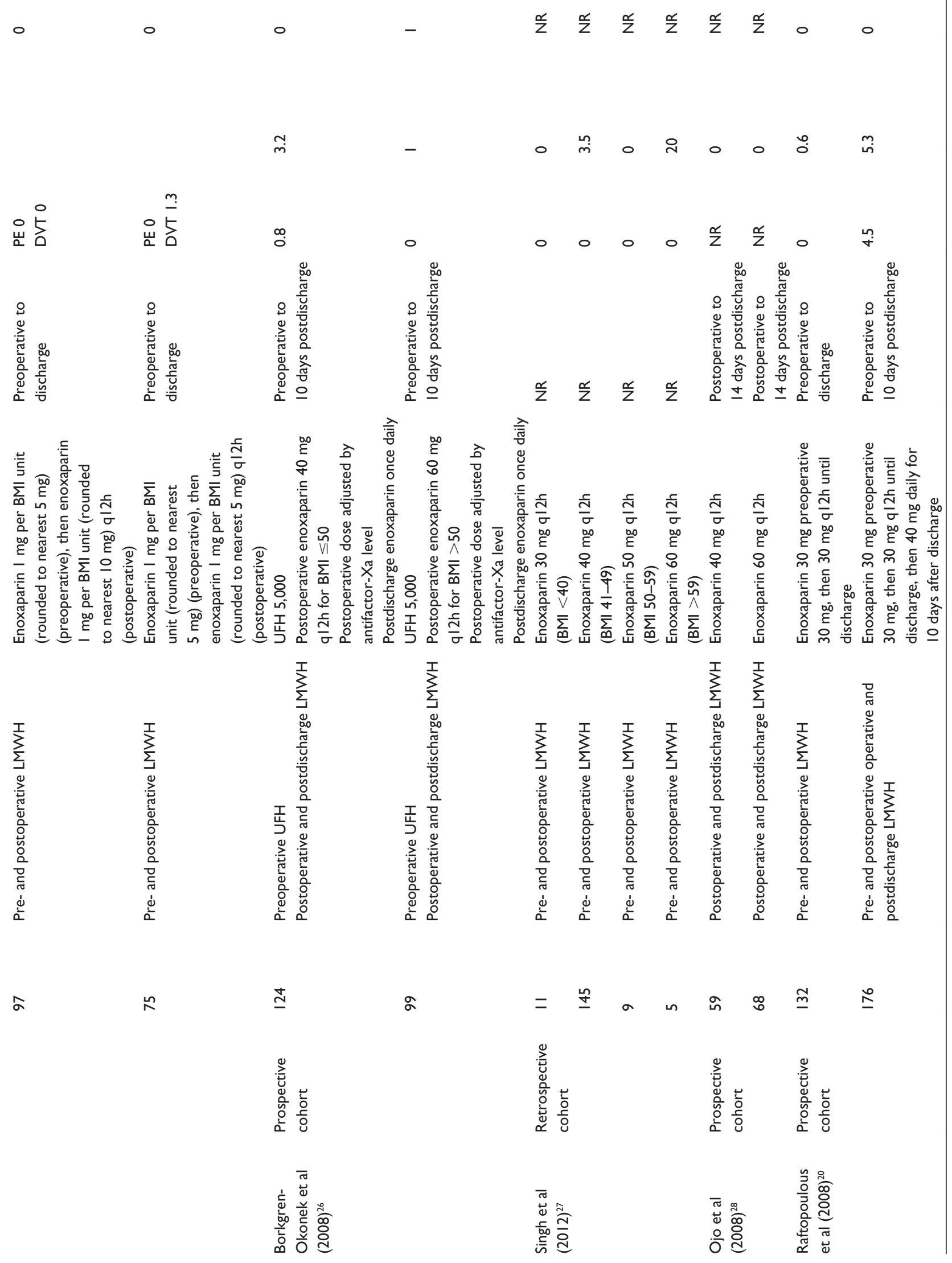


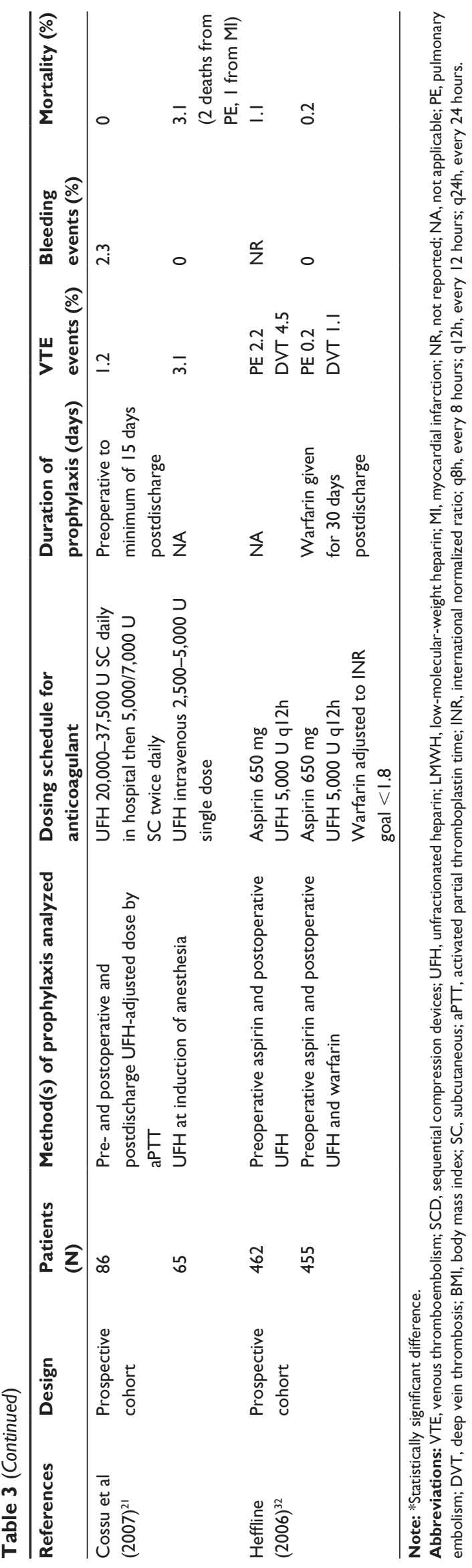

\section{VTE risk assessment and stratification}

Finks et $\mathrm{al}^{9}$ identified patient- and procedure-related VTE risk factors through analysis of the Michigan Bariatric Surgery Collaborative (MBSC) database of over 27,000 patients undergoing bariatric surgery in 32 different hospitals. From their analysis, this group developed a preoperative risk assessment model to stratify bariatric surgery patients by VTE risk and then validated this using a bootstrap method. The following characteristics were included in the model: procedure type, patient history of VTE, male sex, BMI, age, and operative time $>3$ hours. This scheme was able to stratify patients into low-, medium-, and high-risk groups with $<1 \%, 1-4 \%$, and $>4 \% 30$-day VTE event rates, respectively, although approximately $97 \%$ of patients were stratified to the low risk group. Data have not yet been published regarding outcomes using this calculator and any corresponding varied approaches.

\section{Evaluation of strategies to prevent VTE in bariatric surgery patients}

In this section, the literature evaluating different methods of VTE prevention in bariatric surgery patients is reviewed, including mechanical and pharmacologic approaches. Studies in which VCF were used as primary prophylaxis against PE are also reviewed.

\section{Early ambulation}

Early postoperative ambulation is reported as a VTE prevention strategy in the included studies, but is not analyzed for effectiveness in isolation of other methods. The purpose of early ambulation is to reduce venous stasis and thereby mitigate the risk of DVT. Early ambulation was included as an adjunctive method of prophylaxis is most of the studies in this review.

\section{Lower extremity compression}

LEC is also believed to reduce the risk of DVT by decreasing venous stasis. LEC may be provided by elastic stockings (ES), such as graduated compression stockings or by intermittent pneumatic compression (IPC) as with sequential compression devices ( $\mathrm{SCD}$ ).

The search criteria used in this review did not identify any studies comparing LEC to no LEC in the bariatric surgery population; most of the included studies applied some form of LEC. However, LEC has been evaluated in other surgical populations, including general surgery. In a Cochrane review, Sachdeva et $\mathrm{al}^{46}$ reported a $65 \%$ reduction in the risk of postoperative DVT in trials in which graduated compression 


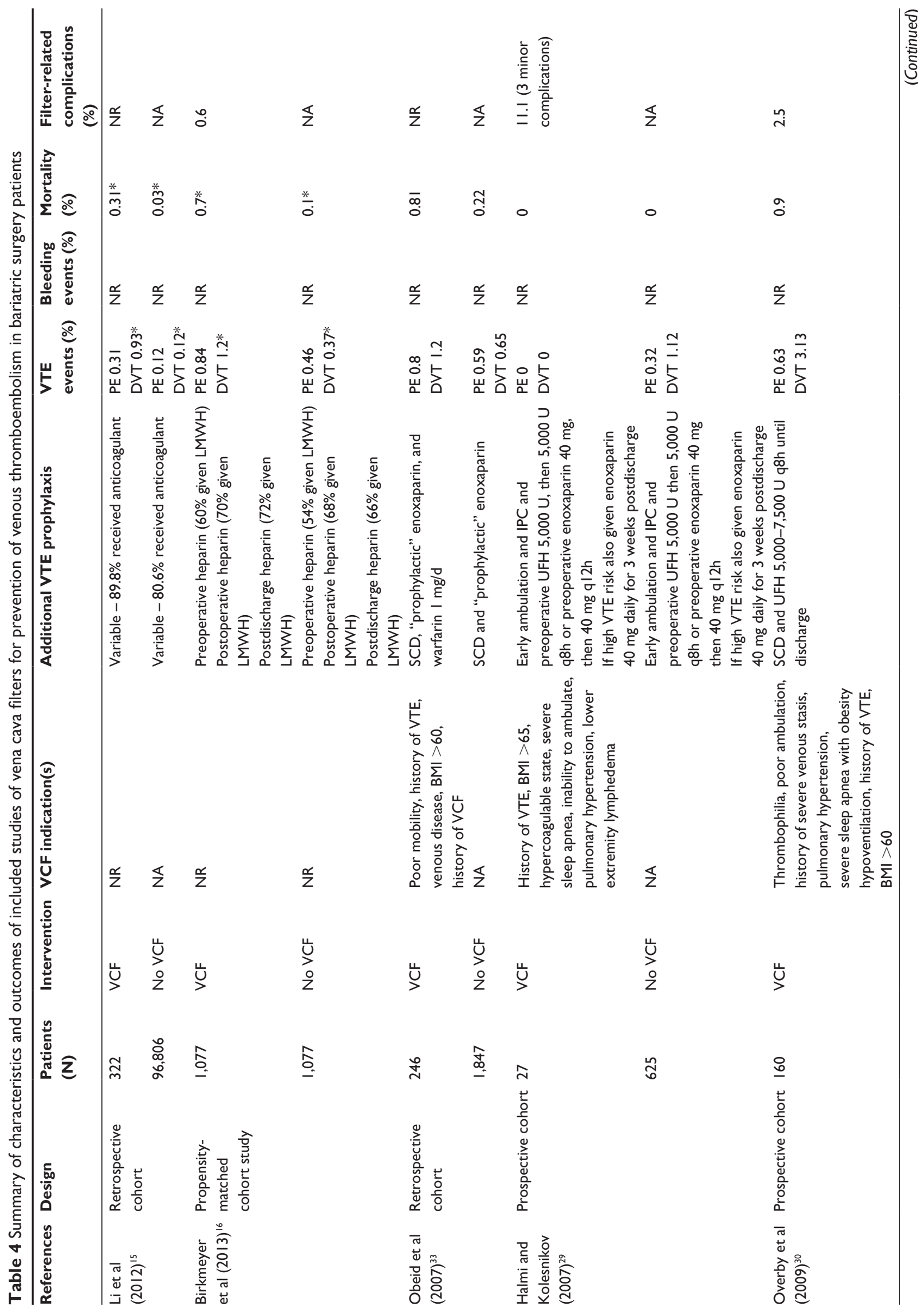




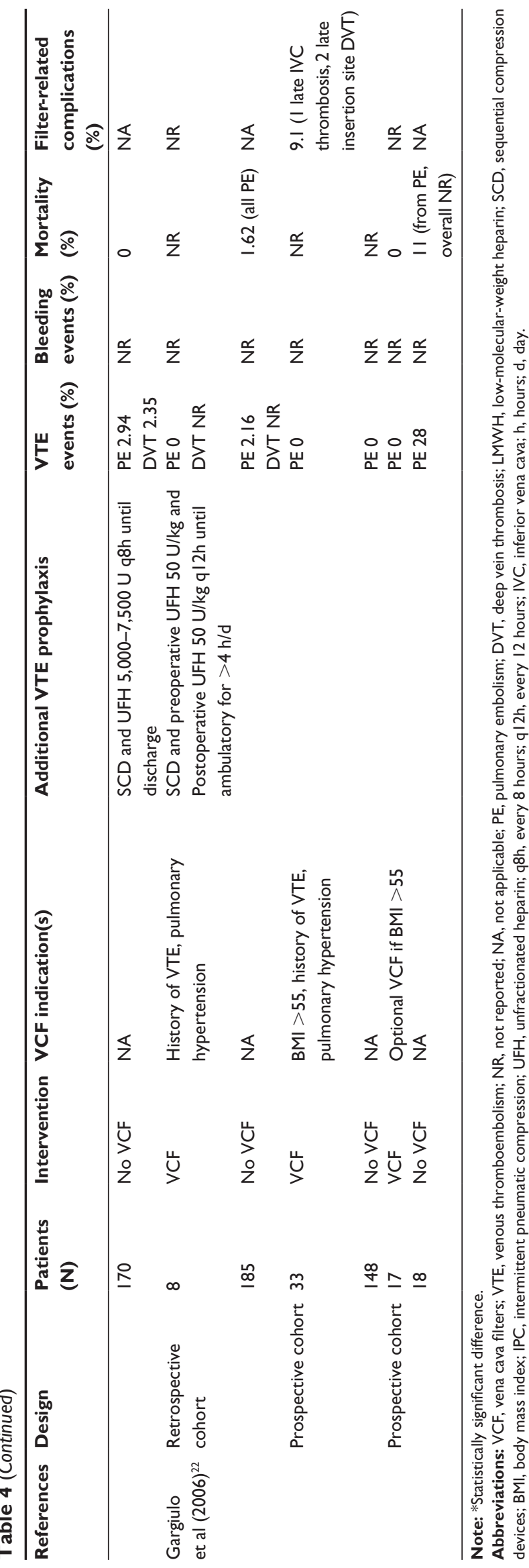

Table 5 Risk factors for venous thromboembolism following bariatric surgery

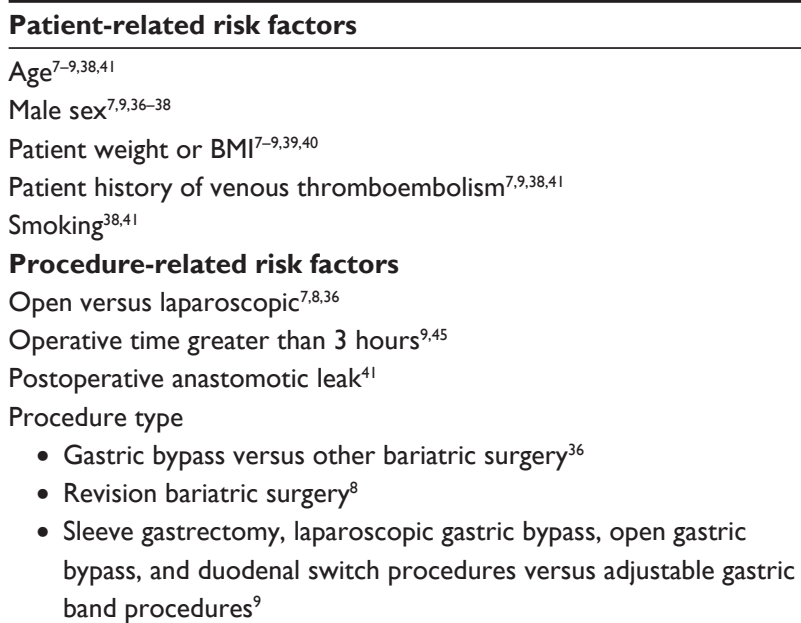

Abbreviation: BMI, body mass index.

stockings were evaluated as VTE prophylaxis. In a metaanalysis of studies comparing IPC with no prophylaxis, the application of IPC appeared to reduce the risk of DVT by approximately $60 \% \cdot{ }^{47}$ Complications of ES may include skin breaks, ulcers, blisters, and skin necrosis. ${ }^{48}$

The favorable risk profile and potential benefits of LEC support its use in postoperative bariatric surgery care.

\section{Pharmacologic prophylaxis}

In bariatric surgical practice, pharmacologic prophylaxis is commonly used. ${ }^{49,50}$ In this section, evaluations of specific medications, their dosing, and duration of use are presented. In these evaluations, the effectiveness of prophylaxis and its safety are both considered since anticoagulants are protective against VTE but have the potential to increase postoperative bleeding.

\section{Comparison of either UFH or LMWH with no pharmacologic prophylaxis}

Direct comparisons of heparins with no pharmacologic VTE prophylaxis after bariatric surgery are limited. Thus, it is useful to consider data from general surgery populations. Multiple studies have evaluated UFH and LMWH for VTE prevention in general surgery and have been the subject of previous reviews. ${ }^{51,52}$ Trials of UFH in surgical patients, including general surgical patients, show risk reductions of $47 \%$ and $41 \%$ for fatal and nonfatal PE, respectively. Data from eight clinical trials in general surgery, in which LMWH was compared with placebo or no prophylaxis, suggest a $71 \%$ risk reduction of overall clinical VTE events and PE (RR, 0.29; CI, 0.11-0.73). ${ }^{51,52}$ These reviews also show that 
the use of either UFH or LMWH may increase postoperative bleeding; major bleeding complications are increased by $57 \%$ across the trials of UFH, and the RR of major hemorrhage with LMWH is 2.03 (CI, 1.37-3.01).

Few studies have compared either UFH or LMWH with no pharmacologic VTE prophylaxis after bariatric surgery. ${ }^{13,17}$ In a multicenter retrospective cohort study, Gagner et $\mathrm{al}^{13}$ found that the 30-day postoperative VTE rates comparing patients receiving combined pharmacologic and SCD to those receiving SCD alone were $0.47 \%$ (CI, 0.30\%-0.74\%) and $0.25 \%$ (CI, $0.04 \%-1.78 \%$ ), respectively. Of note, there were fewer men and fewer sleeve gastrectomy and duodenal switch procedures in the group receiving pharmacologic prophylaxis; the average operative time was longer in the SCD only group. Approximately $90 \%$ of the surgeries in each group were laparoscopic. Mortality within 30 days of surgery was not statistically significantly different between the two groups. Five patients in the SCD plus anticoagulant group required transfusion compared to none in the SCD alone group; the SCD only group was more likely to have blood loss $\geq 50 \mathrm{~mL}$.

Frantzides et $\mathrm{al}^{17}$ compared a universal prophylaxis protocol (all patients given enoxaparin $40 \mathrm{mg}$ SC twice daily and $\mathrm{SCD}$ ) to a risk-stratified protocol (all given SCD, and LMWH was given only for patients with a personal or family history of "hypercoagulable state" or family history of VTE). The risk-stratified group had statistically significantly lower rates of DVT $(0.47 \%$ vs $1.6 \%)$ and PE ( $0 \%$ vs $1.1 \%)$ compared to the universal prophylaxis group. Intraluminal bleeding requiring transfusion was less frequent in the risk-stratified group $(0.4 \%$ vs $4.8 \%)$. Patients in the risk-stratified group had shorter operative times and a lower average BMI. The number of patients in the risk-stratified protocol who received LMWH is not clear, making it difficult to ascertain the impact of restricted use of LMWH. Since the VTE rates are lower in the group with purportedly less LMWH administration, this is an important variable. The dose of LMWH is also higher than standard and could have contributed to bleeding outcomes.

Data comparing pharmacologic prophylaxis with LEC in the bariatric surgery population are limited. Extrapolating from the general surgery literature, pharmacologic prophylaxis for the bariatric surgery patient seems prudent unless the bleeding risk in a specific situation is excessive.

\section{Comparison of UFH with LMWH as pharmacologic prophylaxis}

LMWH has several potential advantages over UFH including high bioavailability, longer half-life, a more predictable anticoagulant response, and lower risk of heparin-induced thrombocytopenia. ${ }^{53,54}$ Multiple studies have compared UFH with LMWH for VTE prevention in general surgery patients. ${ }^{52}$ In a meta-analysis by Mismetti et al, ${ }^{52}$ there was no statistically significant difference between UFH and lowdose LMWH (3,400 anti-Xa units) with respect to clinical PE or DVT outcomes; high-dose LMWH ( $>3,400$ anti-Xa units) was associated with a lower risk of clinical PE, but an increased risk of major hemorrhage.

Only two included studies compared UFH with LMWH for VTE prevention in bariatric surgery. ${ }^{14,18}$ Using the MBSC database, Birkmeyer et $\mathrm{al}^{14}$ compared three prophylaxis regimens; preoperative UFH and postoperative UFH, preoperative UFH and postoperative LMWH, and both pre- and postoperative LMWH, with the former group serving as reference. SCD were used by $98 \%$ of patients and $3.2 \%$ also received a VCF. The groups were compared with mixedeffects logistic regression. Overall, the risk of VTE was $66 \%$ lower in groups receiving LMWH compared with the group receiving postoperative UFH, (OR, 0.34; CI, 0.19-0.62). This difference persisted among subgroups which included only patients at low risk of VTE events (defined as $<1 \%$ ). However, when only patients at high risk of VTE events $(\geq 1 \%$ ) were analyzed, the risk of VTE was not statistically significantly different in groups receiving LMWH compared to UFH (OR, 0.37; CI, 0.11-1.22). Of note, medication doses and duration are not described in this analysis. There was no significant difference across groups with respect to serious hemorrhage, defined as transfusion of $>4$ units of blood product or reoperation for bleeding. Mortality was not reported in this study.

In a nonrandomized study of two consecutive cohorts of 238 patients each, Kothari et $\mathrm{al}^{18}$ compared enoxaparin $40 \mathrm{mg}$ SC twice daily to UFH 5,000 units SC three times daily. All patients received SCD and early ambulation. All underwent laparoscopic RYGB. There were no DVT cases in either group, and only one PE was seen in the UFH group. The average operative time was longer in the UFH group compared to the LMWH group (160 vs 129.5 minutes), but the average BMI was slightly higher in the LMWH group. Postoperative transfusion was given more frequently among the LMWH group compared to the UFH group (5.9\% vs $1.3 \%, P=0.011$ ), and four patients in the LMWH group required reoperation for bleeding. The dose of enoxaparin was higher than standard, potentially influencing the bleeding observations.

The data comparing UFH with LMWH prophylaxis in bariatric surgery are also limited and do not indicate 
superiority of one over another with respect to VTE and bleeding complications. Data from the two described studies do not allow for comparisons of standard heparin doses. Until such data are available, use of either UFH or LMWH appears satisfactory.

\section{Evaluation of adjusted-dose heparin}

Optimal dosing of prophylactic heparin in obese patients such as those undergoing bariatric surgery is unclear. For example, therapeutic LMWH dosing is calculated from total body weight, raising the question as to whether standard prophylactic doses of LMWH are sufficient protection against VTE in obese patients.

Indeed, the majority of studies of pharmacologic prophylaxis in this review evaluated adjusted-dose LMWH after bariatric surgery. ${ }^{19,23-28,31}$ In a multicenter pilot study of two doses of LMWH for prevention of VTE in bariatric surgery, Imberti et $\mathrm{al}^{23}$ randomized 250 subjects to receive either parnaparin 4,250 IU/d (standard prophylactic dose) or $6,400 \mathrm{IU} / \mathrm{d}(150 \%$ of standard dose). Block randomization was stratified by center, patient sex, and BMI. Study medication was given preoperatively and for $9 \pm 2$ days postoperatively, and ascertainment of VTE was conducted only during the time subjects received medication. The groups did not differ with respect to sex, age, BMI, operative time, or other VTE risk factors. The rates of VTE among the standard and adjusted-dose groups were $1.5 \%$ (CI, 0.2-6.0) and $0.8 \%$ (CI, 0.4-5.3), respectively, and were not statistically significantly different. The rates of combined major bleeding and clinically relevant nonmajor bleeding were $6.1 \%$ (CI, 2.9-12.1) and 5\% (CI, 2.1-11.1) for the standard and adjusted-dose groups, respectively. Major bleeding was not reported separately. Being a pilot study, this analysis may not have been adequately powered to detect true difference in effect sizes. In the only other randomized study of pharmacologic prophylaxis in bariatric surgery, Kalfarentzos et $\mathrm{al}^{24}$ compared nadroparin 5,700 IU SC daily (standard prophylactic dose) to 9,500 IU SC daily (167\% of standard dose) in 60 patients undergoing RYGB. There were no VTE events in either group. Given the current knowledge of typical VTE event rates, the small sample size likely did not allow a valid comparison of efficacy. There were two subjects in the higher dose group who developed major bleeding compared with none in the standard-dose group.

Two studies evaluated increased LMWH dosing not based on patient BMI. ${ }^{19,31}$ Scholten et $\mathrm{al}^{19}$ analyzed results from two consecutive groups of patients who were administered different enoxaparin dosing schedules, $30 \mathrm{mg}$ SC twice daily
(92 patients), and $40 \mathrm{mg}$ SC twice daily (389 patients). Most patients underwent open RYGB. All patients reportedly received early ambulation and LEC. Patients with previous VTE were offered postdischarge anticoagulation, but the frequency of this is not reported. Patients in the higher dose group had shorter operative times and length of hospital stay; other reported VTE risk factors were not different between groups. The higher dose group had a lower incidence of VTE events $(0.6 \%$ vs $5.4 \%, P<0.01)$, with no significant difference in bleeding. Of note, there were four PEs in the lower dose group and none in the higher dose group. Hamad and Choban $^{31}$ compared postoperative VTE rates in five hospitals using different LMWH prophylaxis regimens, including one center at which a single preoperative $30 \mathrm{mg}$ dose of enoxaparin was used and another at which enoxaparin $30 \mathrm{mg}$ once daily was given only beginning at discharge. Other centers used $40 \mathrm{mg}$ enoxaparin doses with either once or twice daily dosing frequency. The centers were heterogeneous with respect to the prevalences of VTE risk factors among their patients, and operative times reportedly varied, making valid comparative analysis difficult.

Several cohort studies report results of adjusted-dose LMWH based on patient BMI. ${ }^{25-28}$ Chlysta et $\mathrm{al}^{25}$ compared three regimens of adjusted-dose enoxaparin with respect to thromboembolism, bleeding, and mortality (Table 3). There were significant differences in age, procedure type, and anastomotic leaks between the groups (the latter being more common in the group receiving enoxaparin $40 \mathrm{mg}$ preoperatively). There were no significant differences in VTE or bleeding rates among groups. One PE occurred in the group receiving fixed preoperative enoxaparin dosing. This individual's course was complicated by conversion to open surgery, ventral hernia repair, and wound infection requiring debridement. The authors report that 29 patients were excluded from the analysis as they were not managed by the described protocols. It is possible that these patients were managed differently due to differences in perceived VTE or bleeding risk, introducing bias into the results.

Borkgren-Okonek et $\mathrm{al}^{26}$ compared two different postoperative enoxaparin doses following primary RYGB (93\% laparoscopic) depending on patient BMI (BMI $\leq 50$ given enoxaparin $40 \mathrm{mg}$ twice daily during hospitalization, BMI $>50$ given enoxaparin $60 \mathrm{mg}$ twice daily during hospitalization). All patients received UFH 5,000 units 2 hours preoperatively, IPC, and early ambulation; LMWH was continued once daily for 10 days after discharge. Patients with a known hypercoagulable disorder or a history of VTE were excluded. Antifactor-Xa assays were performed during 
LMWH prophylaxis, with $10 \mathrm{mg}$ dose adjustments made for what were deemed levels out of prophylactic range; of 223 total patients, 37 had dose increases administered. Overall, only one patient (in the enoxaparin $40 \mathrm{mg}$ group) had a VTE event; major bleeding events were noted in five patients (four requiring transfusions and one reoperation) with four bleeds in the $40 \mathrm{mg}$ group. Singh et $\mathrm{al}^{27}$ also analyzed BMI-adjusted LMWH dosing in a cohort of 170 patients undergoing RYGB. All patients received LMWH, 1 hour preoperatively, IPC, and early ambulation. Postoperatively, enoxaparin was given SC twice daily with the following dosing: $\mathrm{BMI} \leq 40,30 \mathrm{mg}$; for BMI 41-49, $40 \mathrm{mg}$; for BMI 50-59 and for BMI $>59,60 \mathrm{mg}$. Overall, there were no VTE events, and five patients had bleeding events, with four of these in the $40 \mathrm{mg}$ group. Of note, 145 of the 170 patients were in the $40 \mathrm{mg}$ group, limiting intergroup comparisons.

Ojo et $\mathrm{al}^{28}$ analyzed results from patients who underwent open RYGB and received either enoxaparin $40 \mathrm{mg}$ SC twice daily or $60 \mathrm{mg}$ SC twice daily, starting in the postoperative period and continuing for 14 days postdischarge. The administered dose was selected by the attending surgeon. Only patients deemed to be at higher risk of VTE were included (personal history of VTE, BMI $\geq 60$, or BMI $\geq 50$ with venous stasis, obstructive sleep apnea, or severe limitation to ambulation). Patients with a history of bleeding or on chronic anticoagulation were excluded. The primary study aim was to ascertain the incidence of major bleeding complications, defined as bleeding during LMWH administration resulting in drug discontinuation, bleeding-related readmission, blood transfusion, or intervention for bleeding. There were no major bleeding events during the 2 week postdischarge study period.

A meta-analysis by Ikesaka et $\mathrm{al}^{55}$ evaluated the efficacy and safety of adjusted-dose heparin in patients undergoing bariatric surgery. Several studies discussed earlier were not included in this meta-analysis likely due to publication date, lack of VTE event reporting, or unclear treatment frequencies. ${ }^{23,25,27,28,31}$ These investigators chose to include two additional studies discussed in this review and one that is not. ${ }^{20,21,56}$ The study by Shepherd et $\mathrm{al}^{56}$ is a single-arm study of LMWH, adjusted daily to a target antifactor-Xa range of $0.1-0.25 \mathrm{U} / \mathrm{mL}$. In the meta-analysis, the rates of in-hospital VTE were $0.54 \%$ (CI, 0.2-1.0) and 2.0\% (CI, 0.1-6.4) for 1,428 adjusted-dose and 430 nonadjusted-dose patients, respectively. For major bleeding, the effect sizes were $1.6 \%$ (CI, 0.6-3.0) and 2.3\% (CI, 1.1-3.9) for those respective groups. Of note, the study by Shepherd et $\mathrm{al}^{56}$ contributed approximately half of the patients in the adjusted-dose group.
Ikesaka et al ${ }^{55}$ used $I^{2}$ as their reported measure of heterogeneity. For the adjusted-dose patient VTE rate, $I^{2}=0 \%$ and for major bleeding, $I^{2}=63.3 \%$, while for non-adjusted-dose patient VTE rate, $I^{2}=71.8 \%$ and for major bleeding, $I^{2}=0 \%$. Variance in effect sizes across studies may be due to either sampling error or to some degree of variance in true effect, depending on the measure and patient group. All $I^{2}$ estimates had wide CIs.

Adjustment of LMWH dose using an antifactor-Xa level was used in two aforementioned studies. ${ }^{26,56} \mathrm{~A}$ detailed discussion regarding the use of antifactor-Xa levels to monitor prophylactic LMWH is beyond the scope of this review, but has been the subject of review elsewhere. ${ }^{57,58}$ There is currently no definitive supportive evidence correlating antifactor-Xa level and postoperative VTE and bleeding risk for bariatric surgery patients.

Concern that standard prophylactic heparin dosing is not optimal in bariatric surgery is reasonable and deserves continued investigation. Currently available data do not support specific strategies for adjusted-dose heparin for VTE prevention in this group. A common design limitation is the lack of comparison of different strategies within groups of patients of similar risk or BMI.

\section{Evaluation of postdischarge heparin prophylaxis}

VTE events may occur in the immediate postoperative period or after hospital discharge. Based on clinical trials, some guidelines endorse consideration of postdischarge prophylaxis in abdominal or pelvic cancer surgery and in major orthopedic surgery. ${ }^{51,59}$ Postdischarge VTE after bariatric surgery is common. ${ }^{5,7}$ For example, Froehling et $\mathrm{al}^{5}$ found that the incidence of VTE rose from $0.3 \%$ to $1.9 \%$ between 7 and 30 days postoperatively. These observations raise the question as to whether the duration of VTE prophylaxis should be extended for bariatric surgery patients.

Two of the included studies specifically evaluated postdischarge pharmacologic prophylaxis in the bariatric surgery population. ${ }^{20,21}$ Other studies in this review included extended duration anticoagulation, but did not compare this practice to standard duration prophylaxis. ${ }^{23,26,28}$ Raftopoulos et $\mathrm{al}^{20}$ compared administration of enoxaparin $30 \mathrm{mg}$ twice daily until hospital discharge only (132 patients) and the addition of enoxaparin $40 \mathrm{mg}$ daily for 10 days following discharge (176 patients). The former group also received enoxaparin $30 \mathrm{mg}$ given 1 hour preoperatively. Of the group who received inhospital prophylaxis only, $4.5 \%$ experienced a VTE event within 30 days of surgery (three patients with PE and three 
with DVT) compared with none in the post-discharge group ( $P=0.006$ for the comparison). Four of the six events in the hospital-only prophylaxis group occurred between hospital discharge and 30 days postoperatively. There was no significant difference in bleeding events between the groups $(0 \%$ vs $4.5 \%, P=0.06)$. One patient in each group required reoperation for bleeding. There were no deaths in either group.

Cossu et $\mathrm{al}^{21}$ compared patients receiving UFH once prior to anesthesia with patients receiving UFH 4-5 days preoperatively and 8-9 days postoperatively, with doses based on activated partial thromboplastin time monitoring, followed by low-dose UFH for at least 15 days after discharge. The authors found a trend toward fewer PE in the extended duration protocol group (1.2\% vs 3\%, $P=$ not significant) but higher rates of bleeding $(2.3 \%$ vs $0 \%$ requiring transfusions, $P=$ not significant). This study essentially compares postoperative UFH prophylaxis with no pharmacologic prophylaxis, making the impact of postdischarge anticoagulation uncertain.

Analysis of postdischarge pharmacologic prophylaxis for bariatric surgery has been limited, but results from Raftopoulos et $\mathrm{al}^{20}$ are promising. Given what is known regarding the timing of postbariatric surgery, longer duration prophylaxis of VTE events merits further evaluation.

\section{Evaluation of oral anticoagulants as VTE prophylaxis}

Oral anticoagulants such as warfarin and other vitamin $\mathrm{K}$ antagonists, direct thrombin inhibitors (dabigatran), and factor Xa inhibitors (rivaroxaban, apixaban, and edoxaban) have been evaluated for VTE prevention after orthopedic surgery, but not in general surgery. ${ }^{60,61}$ We did not identify any studies of oral direct thrombin inhibitors or factor Xa inhibitors for prevention of VTE in bariatric surgery patients. Heffline $^{32}$ described a comparison of a VTE rates in an initial cohort given a combination of UFH and SCD, with a subsequent cohort given a similar regimen except the addition of postoperative warfarin (INR [international normalized ratio] goal of $<1.8$ ). Whether the two cohorts differed with respect to the types of procedures or patient characteristics was not reported. After implementation of the warfarin protocol, VTE events appeared to decrease; statistical significance was not reported. Of note, in the warfarin recipient cohort, patients deemed high risk received VCF, but the number of patients with VCF is not reported. Bleeding events were also not reported.

Given the limited data, oral anticoagulants, including warfarin, are not recommended for VTE prophylaxis in bariatric surgery patients. Neither their efficacy nor associated bleeding risk has been adequately investigated in this patient population.

\section{Evaluation of VCFs as VTE prophylaxis}

$\mathrm{VCF}$ for primary prevention of PE in bariatric surgery patients has been investigated in six studies identified in this review. ${ }^{15,16,22,29,30,33}$

Li et $\mathrm{al}^{15}$ reported the outcomes of 322 patients in the Bariatric Outcomes Longitudinal Database (BOLD) who had preoperative placement of a VCF with those $\sim 97,000$ patients who did not. The presence of multiple patient and procedural characteristics favoring higher risk of VTE were seen with greater frequency in the VCF group, and they were more likely to receive anticoagulation and SCD. Results showed higher rates of DVT $(0.93 \%$ vs $0.12 \%, P=0.001)$ but no statistically significant difference in $\mathrm{PE}(0.31 \%$ vs $0.12 \%)$ when comparing VCF recipients with those who did not have a VCF respectively. All-cause mortality was also higher in the VCF group $(0.31 \%$ vs $0.03 \%, P=0.003)$. Although improved outcomes were not associated with VCF use, the study design allows the possibility that VCF recipients were high-risk patients whose postoperative $\mathrm{PE}$ rate would have been higher without the addition of a VCF.

Birkmeyer et $\mathrm{al}^{16}$ found similar results in an analysis from the MBSC database. Using data from patients who underwent bariatric surgery between 2006 and 2012, Birkmeyer et $\mathrm{al}^{16}$ identified 1,077 propensity matched controls for 1,077 patients who received VCF. These groups did not differ with respect to patient or procedure characteristics, although the VCF group was more likely to receive preoperative LMWH and postdischarge LMWH. Results showed a significantly higher risk of DVT (1.2\% vs $0.4 \%$; OR, $3.3 ; P=0.039)$ in the VCF group and a nonsignificant trend toward higher mortality $(0.7 \%$ vs $0.1 \%$; OR, 7.0 ; $P=0.068)$. There was also a nonsignificant trend toward increased risk of PE in the VCF group $(0.84 \%$ vs $0.46 \%$; OR, 2.0; $P=0.232$ ).

In another registry study, Obeid et $\mathrm{al}^{33}$ reported nonsignificant trends toward higher rates of PE ( $0.8 \%$ vs $0.59 \%)$, DVT $(1.21 \%$ vs $0.65 \%)$, and mortality ( $0.81 \%$ vs $0.22 \%)$ in 246 patients who received a VCF compared to those who did not. VCF recipients were more likely male and had a higher average BMI, but the distribution of procedure type did not differ between groups. Indications for VCF included previous VTE, poor mobility, venous disease, and BMI $>60 \mathrm{~kg} / \mathrm{m}^{2}$. Despite the selection of higher risk patients for VCF, outcomes did not appear to improve with this intervention 
although again, it is unknown whether PE rates would have been higher without VCF.

In contrast, three studies reported more favorable outcomes with VCF. Halmi and Kolesnikov ${ }^{29}$ found a nonsignificant trend toward lower rates of DVT and PE in patients receiving $\mathrm{VCF}$ ( $0 \%$ vs $0.32 \%$ and $0 \%$ vs $7.12 \%$, respectively). Their study reports outcomes for 652 patients undergoing mini-open RYGB. Of these patients, 27 received a VCF based on indications of previous VTE, BMI $>65$, severe sleep apnea, a hypercoagulable state, pulmonary hypertension, lower extremity lymphedema, or inability to ambulate. However, the study reported VCF patients deemed to be at "significant VTE risk" (indications not reported) were given an additional 3 weeks of antithrombotic therapy. Overby et $\mathrm{al}^{30}$ published an experience with 330 patients undergoing primary RYGB in which high risk patients received a VCF. High risk was defined as the presence of a thrombophilia, BMI $>60$, history of VTE, severe venous stasis or severe sleep apnea with obesity-hypoventilation, poor ambulation, or pulmonary hypertension. In this analysis, VCF recipients had lower rates of $\mathrm{PE}(0.63 \%$ vs $2.94 \%)$ but higher rates of DVT (3.13\% vs 2.35\%). Neither of these differences reached statistical significance.

Gargiulo et $\mathrm{al}^{22}$ describe results of three practice patterns for VTE prevention after open RYGB (Table 4). In a retrospective review, they noted that $\mathrm{PE}$ occurred in patients without VCF who had BMI $>55$. In the subsequent period in which $\mathrm{BMI}>55$ was added to the indications for VCF placement, no patients with BMI $>55$ and a VCF had postoperative $\mathrm{PE}$. In a third period during which patients with a BMI of $>55$ were offered optional VCF placement, the incidence of PE $(0 \%$ vs $28 \%, P<0.05)$ and PE-specific mortality $(0 \%$ vs $11 \%, P<0.05)$ appeared to be less with VCF. These small groups did not appear to differ on other reported risk factors for VTE, but the process by which patients opted in for VCF placement was not described.

Complications directly related to VCF include strut fracture, filter migration, vena cava perforation, VCF thrombosis, and insertion site infection or thrombosis. ${ }^{62} \mathrm{VCF}$ migration may involve serious injury to heart valves, the myocardium, or pericardium. Although uncommon, understanding the risk of these complications is important when VCF placement is being considered. Among the included studies, four reported VCF-related complications..$^{16,22,29,30}$ For example, in the analysis by Overby et al, ${ }^{30}$ complications included pneumothorax, filter migration to the right atrium, hemopericardium, and inability to ablate an accessory pathway in atrial fibrillation due to the filter. In their analysis, Birkmeyer et $\mathrm{al}^{16}$ reported
VCF complications including a damaged heart valve requiring replacement, two fatal inferior vena cava thrombi, contrast nephropathy, and an incision site infection. Retrieval of VCF was reported in two studies, with success rates $>90 \%$ in both. ${ }^{29,30}$

There have been two published systematic reviews with meta-analyses addressing the use of VCF in bariatric surgery. ${ }^{63,64}$ Both included the studies discussed earlier with the exception of the study by Hamli and Kolesnikov, ${ }^{29}$ which was not included by Brotman et al. ${ }^{63}$ Both metaanalyses indicate that VCF do not appear to decrease the risk of PE. The RR of PE determined by Kaw et a ${ }^{64}$ was 1.02 (CI, 0.31-3.37) and by Brotman et al ${ }^{63} 1.21$ (CI, 0.572.56). VCF did appear to increase the risk of postoperative DVT by both meta-analyses; Brotman et $\mathrm{al}^{63}$ found a RR of 2.94 (CI, 1.35-6.38) and Kaw et al ${ }^{64}$ a RR of 2.81 (CI, $1.33-5.97)$. Brotman et $\mathrm{l}^{63}$ found a significantly increased risk of mortality among recipients of VCF across their included studies (RR, 4.30; CI, 1.60-11.54), while Kaw et $\mathrm{al}^{64}$ noted a nonsignificant trend toward higher mortality rates among VCF recipients (RR, 3.27; CI, 0.78-13.64). Studies by Hamli and Kolesnikov ${ }^{29}$ and Overby et $\mathrm{al}^{30}$ were not included in the former mortality analysis.

In the meta-analysis by Kaw et $\mathrm{al}^{64}$ the only reported measure of heterogeneity is $I^{2}$. For the estimated effect sizes, $I^{2}$ ranged from $35 \%$ to $60 \%$, suggesting that a proportion of the observed variance in effect sizes may reflect true differences across studies. Brotman et $\mathrm{a}^{63}$ found a similar $I^{2}$ result for DVT risk ratio (40.3\%), but for PE and mortality effect sizes the $I^{2}$ were $6.9 \%$ and $0 \%$, respectively, suggesting that for those measures, most or all of the observed variance is due to sampling error.

Available data do not appear to support the routine placement of VCF as an adjunctive method of prophylaxis in bariatric surgery. Currently, all the available data on VCF placement is observational in nature, which limits the ability to accurately determine efficacy of an intervention; randomized controlled trials are needed for this.

\section{Selected published guideline recommendations}

Several organizations or professional societies have published guideline recommendations for prevention of VTE in bariatric surgery patients (Table 6). ${ }^{51,65-67}$ None of the published guidelines reviewed discusses different approaches to VTE prevention based on procedure type, BMI, or open versus laparoscopic surgeries. Guidelines do reflect the uncertainties in the literature discussed. 


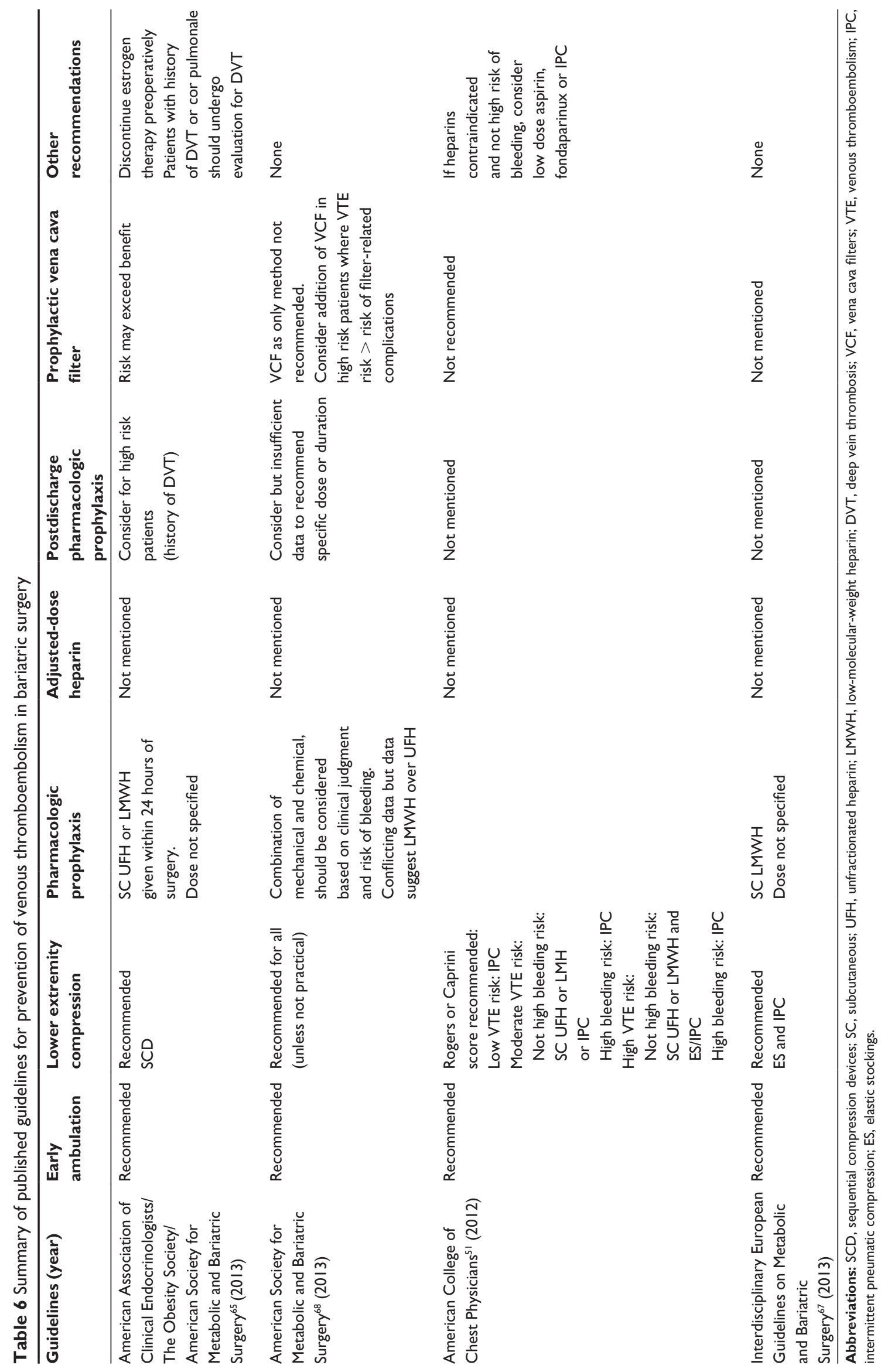


The American Association of Clinical Endocrinologists, the Obesity Society, and the American Society for Metabolic and Bariatric Surgery (AACE/TOS/ASMBS) together have produced guidelines for bariatric surgery. ${ }^{65}$ Early ambulation and IPC as well as postoperative UFH or LMWH are recommended. Anticoagulant dosing is not specified, but postdischarge pharmacologic prophylaxis is recommended for "high-risk" patients, such as those with history of DVT; no specific duration of therapy is suggested. These organizations discuss that VCF may present a greater risk than benefit due to filter-related complications. They also recommend preoperative discontinuation of estrogen medications (one cycle of oral contraceptives and 3 weeks for hormone replacement therapy) since these may increase the risk of VTE. Finally, they recommend preoperative DVT screening for patients with a history of DVT or cor pulmonale; evidence supporting this consideration is not discussed.

The ASMBS provides a separate set of recommendations. ${ }^{68}$ The ASMBS recommends prophylaxis with a combination of early ambulation and mechanical prophylaxis for all patients. They state that use of pharmacologic prophylaxis "should be considered based on clinical judgment and risk of bleeding". The ASMBS expresses a preference for LMWH over UFH, although they stipulate that there is conflicting data regarding the type of pharmacologic prophylaxis to use. The ASMBS also recommends extended duration of pharmacologic prophylaxis but do not provide specific dose or duration recommendations. Although VCF are not recommended as the only method of prophylaxis, addition of VCF to mechanical and pharmacologic prophylaxis may be considered in selected high-risk patients for whom the risk of VTE outweighs the risk of filter-related complications.

The American College of Chest Physicians (ACCP) does not offer specific recommendations for bariatric surgery patients, but includes this group with patients having other abdominal, vascular, or plastic reconstructive surgery. ${ }^{51}$ The ACCP recommends using either a Rogers et $a l^{35}$ or Caprini ${ }^{34}$ score to stratify patients as low, moderate, or high risk of VTE. Given usual body habitus and type of surgery, bariatric surgery patients typically will be considered moderate to high VTE risk, depending on comorbidities. The ACCP also recommends stratifying bleeding risk to determine a prophylaxis plan. For patients with a moderate VTE risk who are not considered to be at high bleeding risk, LMWH, UFH, or IPC may be used; for moderate VTE risk but high bleeding risk, IPC alone is recommended. For patients with a high VTE risk, a combination of mechanical (IPC or ES) and pharmacologic (either LMWH or UFH) measures are recommended, unless the bleeding risk is high (IPC alone is recommended). The ACCP also recommends against the use of VCF for primary prophylaxis and does not offer specific recommendations regarding adjusted dose heparins or extended duration anticoagulation postdischarge.

The Interdisciplinary European Guidelines on Metabolic Surgery recommend VTE prevention for all bariatric patients through LMWH administration, use of LEC (both ES and SCD), and early postoperative ambulation. ${ }^{67}$ These guidelines do not address the questions of augmented LMWH dosing, postdischarge anticoagulation, or VCF placement.

Published guidelines have in common the recommendation for early ambulation and LEC and generally concur regarding the use of heparin prophylaxis. However, the uncertainties regarding adjusted-dose and post-discharge heparin are reflected in the guidelines. They are also generally concurrent in their recommendations regarding prophylactic VCF.

\section{Conclusion and summary recommendations}

VTE continues to be an important source of postoperative morbidity and mortality among patients undergoing bariatric surgery, despite current VTE prevention methods. The practice of postbariatric surgery VTE prophylaxis has been primarily supported by data from the general surgery literature. Postoperative care encouraging early ambulation, use of LEC, and pharmacologic prophylaxis (assuming satisfactory bleeding risk) appears prudent. To date, the literature regarding further optimization of preventive approaches specific to bariatric surgery patients has been limited. However, the principles behind adjusted-dose heparin and post-discharge prophylaxis are worthy of further analysis with randomized controlled trials to assess their efficacy and safety. At present, VCF do not have an established role in bariatric surgery VTE prophylaxis. Studies are also needed which better control for patient and procedure-related VTE risk factors, and future studies may incorporate what is known regarding patient and procedure-related risk factors to develop validated, stratified management plans using different intensities of prophylaxis. Until more data are available, institutional quality improvement efforts should focus on ensuring consistent application of established methods.

\section{Disclosure}

The authors report no conflicts of interest in this work. 


\section{References}

1. Buchwald H, Avidor Y, Braunwald E, et al. Bariatric surgery: a systematic review and meta-analysis. JAMA. 2004;292(14):1724-1737.

2. Maggard MA, Shugarman LR, Suttorp M, et al. Meta-analysis: surgical treatment of obesity. Ann Intern Med. 2005;142(7):547-559.

3. Colquitt JL, Pickett K, Loveman E, Frampton GK. Surgery for weight loss in adults. Cochrane Database Syst Rev. 2014;8:CD003641.

4. Stein PD, Matta F. Pulmonary embolism and deep venous thrombosis following bariatric surgery. Obes Surg. 2013;23(5):663-668.

5. Froehling DA, Daniels PR, Mauck KF, et al. Incidence of venous thromboembolism after bariatric surgery: a population-based cohort study. Obes Surg. 2013;23(11):1874-1879.

6. Lancaster RT, Hutter MM. Bands and bypasses: 30-day morbidity and mortality of bariatric surgical procedures as assessed by prospective, multi-center, risk-adjusted ACS-NSQIP data. Surg Endosc. 2008;22(12):2554-2563.

7. Winegar DA, Sherif B, Pate V, Demaria EJ. Venous thromboembolism after bariatric surgery performed by Bariatric Surgery Center of Excellence Participants: analysis of the Bariatric Outcomes Longitudinal Database. Surg Obes Relat Dis. 2011;7(2):181-188.

8. Jamal MH, Corcelles R, Shimizu H, et al. Thromboembolic events in bariatric surgery: a large multi-institutional referral center experience. Surg Endosc. 2015;29(2):376-380.

9. Finks JF, English WJ, Carlin AM, et al. Predicting risk for venous thromboembolism with bariatric surgery: results from the Michigan bariatric surgery collaborative. Ann Surg. 2012;255(6):1100-1104.

10. Sapala JA, Wood MH, Schuhknecht MP, Sapala MA. Fatal pulmonary embolism after bariatric operations for morbid obesity: a 24-year retrospective analysis. Obes Surg. 2003;13(6):819-825.

11. Morino M, Toppino M, Forestieri P, Angrisani L, Allaix ME, Scopinaro N. Mortality after bariatric surgery: analysis of 13,871 morbidly obese patients from a National Registry. Ann Surg. 2007; 246(6):1002-1007.

12. Melinek J, Livingston E, Cortina G, Fishbein MC. Autopsy findings following gastric bypass surgery for morbid obesity. Arch Pathol Lab Med. 2002;126(9):1091-1095.

13. Gagner M, Selzer F, Belle SH, et al. Adding chemoprophylaxis to sequential compression might not reduce risk of venous thromboembolism in bariatric surgery patients. Surg Obes Relat Dis. 2012;8(6):663-670.

14. Birkmeyer NJ, Finks JF, Carlin AM, et al. Comparative effectiveness of unfractionated and low-molecular-weight heparin for prevention of venous thromboembolism following bariatric surgery. Arch Surg. 2012;147(11):994-998.

15. Li W, Gorecki P, Semaan E, Briggs W, Tortolani AJ, D’Ayala M. Concurrent prophylactic placement of inferior vena cava filter in gastric bypass and adjustable banding operations in the Bariatric Outcomes Longitudinal Database. J Vasc Surg. 2012;55(6):1690-1695.

16. Birkmeyer NJ, Finks JF, English WJ, et al. Risks and benefits of prophylactic inferior vena cava filters in patients undergoing bariatric surgery. J Hosp Med. 2013;8(4):173-177.

17. Frantzides CT, Welle SN, RuffTM, Frantzides AT. Routine anticoagulation for venous thromboembolism prevention following laparoscopic gastric bypass. JSLS. 2012;16(1):33-37.

18. Kothari SN, Lambert PJ, Mathiason MA. A comparison of thromboembolic and bleeding events following laparoscopic gastric bypass in patients treated with prophylactic regimens of unfractionated heparin or enoxaparin. Am J Surg. 2007;194(6):709-711.

19. Scholten DJ, Hoedema RM, Scholten SE. A comparison of two different prophylactic dose regimens of low molecular weight heparin in bariatric surgery. Obes Surg. 2002;12(1):19-24.

20. Raftopoulos I, Martindale C, Cronin A, Steinberg J. The effect of extended post-discharge chemical thromboprophylaxis on venous thromboembolism rates after bariatric surgery: a prospective comparison trial. Surg Endosc. 2008;22(11):2384-2391.

21. Cossu ML, Pilo L, Piseddu G, Tilocca PL, Cossu F, Noya G. Prophylaxis of venous thromboembolism in bariatric surgery. Chir Ital. 2007;59(3):331-335.
22. Gargiulo IN, Veith FJ, Lipsitz EC, Suggs WD, Ohki T, Goodman E. Experience with inferior vena cava filter placement in patients undergoing open gastric bypass procedures. J Vasc Surg. 2006; 44(6):1301-1305.

23. Imberti D, Baldini E, Pierfranceschi MG, et al. Prophylaxis of venous thromboembolism with low molecular weight heparin in bariatric surgery: a prospective, randomised pilot study evaluating two doses of parnaparin (BAFLUX study). Obes Surg. 2014;24(2):284-291.

24. Kalfarentzos F, Stavropoulou F, Yarmenitis S, et al. Prophylaxis of venous thromboembolism using two different doses of low-molecularweight heparin (nadroparin) in bariatric surgery: a prospective randomized trial. Obes Surg. 2001;11(6):670-676.

25. Chlysta WJ, Iffland PH, Haller NA. Review of BMI-based pharmacologic protocols for the prevention of venous thromboembolism in bariatric surgery patients. Bariatric Surg Pract Patient Care. 2014;9(3):91-96.

26. Borkgren-Okonek MJ, Hart RW, Pantano JE, et al. Enoxaparin thromboprophylaxis in gastric bypass patients: extended duration, dose stratification, and antifactor Xa activity. Surg Obes Relat Dis. 2008;4(5):625-631.

27. Singh K, Podolsky ER, Um S, et al. Evaluating the safety and efficacy of BMI-based preoperative administration of low-molecular-weight heparin in morbidly obese patients undergoing Roux-en-Y gastric bypass surgery. Obes Surg. 2012;22(1):47-51.

28. Ojo P, Asiyanbola B, Valin E, Reinhold R. Post discharge prophylactic anticoagulation in gastric bypass patient - how safe? Obes Surg. 2008;18(7):791-796.

29. Halmi D, Kolesnikov E. Preoperative placement of retreivable inferior vena cava filters in bariatric surgery. Surg Obes Relat Dis. 2007;3(6):602-605.

30. Overby DW, Kohn GP, Cahan MA, et al. Risk-group targeted inferior vena cava filter placement in gastric bypass patients. Obes Surg. 2009; 19(4):451-455.

31. Hamad GG, Choban PS. Enoxaparin for thromboprophylaxis in morbidly obese patients undergoing bariatric surgery: findings of the prophylaxis against VTE outcomes in bariatric surgery patients receiving enoxaparin (PROBE) study. Obes Surg. 2005;15(10):1368-1374.

32. Heffline MS. Preventing vascular complications after gastric bypass. J Vasc Nurs 2006;24(2):50-54.

33. Obeid FN, Bowling WM, Fike JS, Durant JA. Efficacy of prophylactic inferior vena cava filter placement in bariatric surgery. Surg Obes Relat Dis. 2007;3(6):606-608.

34. Caprini JA. Risk assessment as a guide for the prevention of the many faces of venous thromboembolism. Am J Surg. 2010;199(1 Suppl): S3-S10.

35. Rogers SO Jr, Kilaru RK, Hosokawa P, Henderson WG, Zinner MJ, Khuri SF. Multivariable predictors of postoperative venous thromboembolic events after general and vascular surgery: results from the patient safety in surgery study. J Am Coll Surg. 2007;204(6):1211-1221.

36. Masoomi H, Buchberg B, Reavis KM, Mills SD, Stamos M, Nguyen NT. Factors predictive of venous thromboembolism in bariatric surgery. Am Surg. 2011;77(10):1403-1406.

37. Livingston EH, Huerta S, Arthur D, Lee S, De Shields S, Heber D. Male gender is a predictor of morbidity and age a predictor of mortality for patients undergoing gastric bypass surgery. Ann Surg. 2002; 236(5):576-582.

38. Steele KE, Schweitzer MA, Prokopowicz G, et al. The long-term risk of venous thromboembolism following bariatric surgery. Obes Surg. 2011;21(9):1371-1376.

39. Carmody BJ, Sugerman HJ, Kellum JM, et al. Pulmonary embolism complicating bariatric surgery: detailed analysis of a single institution's 24-year experience. J Am Coll Surg. 2006;203(6):831-837.

40. Abou-Nukta F, Alkhoury F, Arroyo K, et al. Clinical pulmonary embolus after gastric bypass surgery. Surg Obes Relat Dis. 2006;2(1):24-28.

41. Gonzalez R, Haines K, Nelson LG, Gallagher SF, Murr MM. Predictive factors of thromboembolic events in patients undergoing Roux-en-Y gastric bypass. Surg Obes Relat Dis. 2006;2(1):30-35.

42. Nguyen NT, Owings JT, Gosselin R, et al. Systemic coagulation and fibrinolysis after laparoscopic and open gastric bypass. Arch Surg. 2001;136(8):909-916. 
43. Overby DW, Kohn GP, Cahan MA, et al. Prevalence of thrombophilias in patients presenting for bariatric surgery. Obes Surg. 2009;19(9):1278-1285.

44. Taura P, Rivas E, Martinez-Palli G, et al. Clinical markers of the hypercoagulable state by rotational thrombelastometry in obese patients submitted to bariatric surgery. Surg Endosc. 2014;28(2):543-551.

45. Chan MM, Hamza N, Ammori BJ. Duration of surgery independently influences risk of venous thromboembolism after laparoscopic bariatric surgery. Surg Obes Relat Dis. 2013;9(1):88-93.

46. Sachdeva A, Dalton M, Amaragiri SV, Lees T. Elastic compression stockings for prevention of deep vein thrombosis. Cochrane Database Syst Rev. 2010;(7):CD001484.

47. Urbankova J, Quiroz R, Kucher N, Goldhaber SZ. Intermittent pneumatic compression and deep vein thrombosis prevention. A meta-analysis in postoperative patients. Thromb Haemost. 2005;94(6):1181-1185.

48. The CLOTS Trials Collaboration. Effectiveness of thigh-length graduated compression stockings to reduce the risk of deep vein thrombosis after stroke (CLOTS trial 1): a multicentre, randomised controlled trial Lancet. 2009;373(9679):1958-1965.

49. Pryor HI, Singleton A, Lin E, Lin P, Vaziri K. Practice patterns in high-risk bariatric venous thromboembolism prophylaxis. Surg Endosc. 2013;27(3):843-848.

50. Barba CA, Harrington C, Loewen M. Status of venous thromboembolism prophylaxis among bariatric surgeons: have we changed our practice during the past decade? Surg Obes Relat Dis. 2009;5(3):352-356.

51. Gould MK, Garcia DA, Wren SM, et al. Prevention of VTE in nonorthopedic surgical patients. Antithrombotic Therapy and Prevention of Thrombosis, 9th ed: American College of Chest Physicians Evidence-Based Clinical Practice Guidelines. Chest. 2012;141 (2 Suppl):e227S-e277S.

52. Mismetti P, Laporte S, Darmon JY, Buchmüller A, Decousus H. Meta-analysis of low molecular weight heparin in the prevention of venous thromboembolism in general surgery. Br J Surg. 2001;88(7): 913-930

53. Weitz JI. Low-molecular-weight heparins. N Engl J Med. 1997;337(10): 688-699.

54. Garcia DA, Baglin TP, Weitz JI, Samama MM. Parenteral anticoagulants: Antithrombotic Therapy and Prevention of Thrombosis, 9th ed: American College of Chest Physicians Evidence-Based Clinical Practice Guidelines. Chest. 2012;141(2 Suppl):e24S-e43S.

55. Ikesaka R, Delluc A, Le Gal G, Carrier M. Efficacy and safety of weight-adjusted heparin prophylaxis for the prevention of acute venous thromboembolism among obese patients undergoing bariatric surgery: a systematic review and meta-analysis. Thromb Res. 2014;133(4): 682-687.

56. Shepherd MF, Rosborough TK, Schwartz ML. Heparin thromboprophylaxis in gastric bypass surgery. Obes Surg. 2003;13(2):249-253.
57. Egan G, Ensom MH. Measuring anti-factor xa activity to monitor low-molecular-weight heparin in obesity: a critical review. Can J Hosp Pharm. 2015;68(1):33-47.

58. Nutescu EA, Spinler SA, Wittkowsky A, Dager WE. Low-molecularweight heparins in renal impairment and obesity: available evidence and clinical practice recommendations across medical and surgical settings. Ann Pharmacother. 2009;43(6):1064-1083.

59. Falck-Ytter Y, Francis CW, Johanson NA, et al. Prevention of VTE in orthopedic surgery patients: Antithrombotic Therapy and Prevention of Thrombosis, 9th ed: American College of Chest Physicians Evidence-Based Clinical Practice Guidelines. Chest. 2012;141 (2 Suppl):e278S-e325S.

60. Messerschmidt C, Friedman RJ. Clinical experience with novel oral anticoagulants for thromboprophylaxis after elective hip and knee arthroplasty. Arterioscler Thromb Vasc Biol. 2015;35(4):771-778.

61. Samama C, Vray M, Barré J, et al. Extended venous thromboembolism prophylaxis after total hip replacement: a comparison of lowmolecular-weight heparin with oral anticoagulant. Arch Intern Med. 2002;162(19):2191-2196.

62. Rajasekhar A, Streiff MB. Vena cava filters for management of venous thromboembolism: a clinical review. Blood Rev. 2013;27(5): 225-241.

63. Brotman DJ, Shihab HM, Prakasa KR, et al. Pharmacologic and mechanical strategies for preventing venous thromboembolism after bariatric surgery: a systematic review and meta-analysis. JAMA Surg. 2013;148(7):675-686.

64. Kaw R, Pasupuleti V, Wayne Overby D, et al. Inferior vena cava filters and postoperative outcomes in patients undergoing bariatric surgery: a meta-analysis. Surg Obes Relat Dis. 2014;10(4):725-733.

65. Mechanick JI, Youdim A, Jones DB, et al. Clinical practice guidelines for the perioperative nutritional, metabolic, and nonsurgical support of the bariatric surgery patient-2013 update: cosponsored by American Association of Clinical Endocrinologists, The Obesity Society, and American Society for Metabolic and Bariatric Surgery. Obesity. 2013; 21(Suppl 1):S1-S27.

66. ASMBS updated position statement on prophylactic measures to reduce the risk of venous thromboembolism in bariatric surgery patients. Surg Obes Relat Dis. 2013;9(4):493-497.

67. Fried M, Yumuk V, Oppert JM, et al. Interdisciplinary European Guidelines on metabolic and bariatric surgery. Obes Facts. 2013; 6(5):449-468.

68. Brethauer SA. ASMBS updated position statement on prophylactic measures to reduce the risk of venous thromboembolism in bariatric surgery patients. Surg Obes Relat Dis. 2013;9(4):493-497.
Vascular Health and Risk Management

\section{Publish your work in this journal}

Vascular Health and Risk Management is an international, peerreviewed journal of therapeutics and risk management, focusing on concise rapid reporting of clinical studies on the processes involved in the maintenance of vascular health; the monitoring, prevention and treatment of vascular disease and its sequelae; and the involvement of

\section{Dovepress}

metabolic disorders, particularly diabetes. This journal is indexed on PubMed Central and MedLine. The manuscript management system is completely online and includes a very quick and fair peer-review system, which is all easy to use. Visit http://www.dovepress.com/ testimonials.php to read real quotes from published authors. 